\title{
Evaluation of Rapid Diagnostic Tests for Early Diagnosis of Malaria in Rural India to Prevent Deadly Complications
}

\author{
V.S. Prasad ${ }^{1}$, V. Sindhura², M.N. Kumar ${ }^{3}$
}

\begin{abstract}
${ }^{1}$ Department of Family Medicine, Padmaja Hospital, Vizianagaram, Andhra Pradesh, India. ${ }^{2}$ Department of Paediatrics, King George Hospital, Visakhapatnam, Andhra Pradesh, India. ${ }^{3}$ Department of Plastic Surgery, Visakha Institute of

Medical Sciences, Visakhapatnam, Andhra Pradesh, India.
\end{abstract}

\section{ABSTRACT}

\section{BACKGROUND}

As per World Malaria Report, 2018, There were an estimated 219 million cases and 435000 related deaths in 2017, with about 9.5 million malaria cases and 16,733 related deaths in 2017 in India. The most severe clinical form of malaria is caused by $P$. falciparum infection. The disease is usually fatal in non-immunes. The complications of falciparum malaria are cerebral malaria, pulmonary oedema, septicaemia and shock. If these complications are not diagnosed early and are not treated adequately, the patient becomes fatal. To prevent these complications, we need early diagnosis. Clinical diagnosis of malaria requires microscopic detection of the parasite. Diagnosis of malaria by microscopy is the gold standard but requires expertise and is time consuming. Recently, new rapid malaria detection tests based on detection of an enzyme produced by live parasites, parasite lactate dehydrogenase (pLDH) and Histidine Rich Proteins (HRPII) have been developed. It is simple, easy to use and does not require expertise. In the present study, the Rapid Diagnostic Tests are evaluated for the effectiveness for early diagnosis of Malaria in Rural India to prevent deadly complications.

\section{METHODS}

The study, conducted in a hospital, included 733 patients with fever of 3 days duration after obtaining consent from the subjects and approval from the ethical committee. 733 whole blood samples were collected and tested for Smear Microscopy for Malarial parasites and were also tested with Rapid Diagnostic Tests. The study was spread over 10 months from June, 2018 to March, 2019 and results were sent for analysis. Rapid Diagnostic Test (RDT) was compared with 'Gold Standard' technique i.e.; Microscopy- Smear Examination with the help of $\mathrm{X}^{2}$ values, sensitivity, specificity, positive predictive values, negative predictive values, percentage of false negatives, percentage of false positives, positive likelihood ratio(LR+), and negative likelihood ratio (LR-) values.

\section{RESULTS}

The results of Rapid Diagnostic Test (RDT) were compared with smear microscopy and it was found that RDT is as effective as Smear Microscopy in diagnosing Malaria with $97.81 \%$ sensitivity, $97.82 \%$ specificity, 97.20\% Positive predictive value and $98.30 \%$ Negative predictive value. In diagnosing the species of Malaria also, RDT is found to be as effective as Smear Microscopy in differentiating Falciparum Malaria with $97.98 \%$ sensitivity value, $98.79 \%$ specificity value, $97.49 \%$ positive predictive value, $99.03 \%$ negative predictive value. RDT is found to be as effective as Smear Microscopy in differentiating Vivax Malaria with $97.54 \%$ sensitivity, 99.03\% specificity, $96.75 \%$ positive predictive value and $99.27 \%$ negative predictive value.

\section{CONCLUSIONS}

The present study concludes Rapid Diagnostic Tests are effective screening tests as shown by the high sensitivity, specificity and predictive values, high Likelihood positive(LR+) and low Likelihood Negative(LR-) values.

\section{KEY WORDS}

Smear for Malarial Parasite, QBC Test, Rapid Diagnostic Test, Falciparum Malaria, Vivax Malaria, Cerebral Malaria

\author{
Corresponding Author: \\ Dr. Vinnakota Sindhura, \\ Flat No. 102, ELRS Residency, \\ Krishna Nagar, Maharani Peta, \\ Visakhapatnam-530002, \\ Andhra Pradesh, India. \\ E-mail: drsindhura.vinnakota@gmail.com
}

DOI: $10.14260 /$ jemds/2019/739

Financial or Other Competing Interests: None.

How to Cite This Article:

Prasad VS, Sindhura V, Kumar MN. Evaluation of rapid diagnostic tests for early diagnosis of malaria in rural India to prevent deadly complications. J. Evolution Med. Dent. Sci. 2019;8(45):3408-3412, DOI: 10.14260/jemds/2019/739

Submission 19-07-2019,

Peer Review 25-10-2019,

Acceptance 31-10-2019,

Published 11-11-2019. 


\section{BACKGROUND}

As per World Malaria Report 2018, there were an estimated 219 million cases and 435000 related deaths in 2017,1 with about 9.5 million malaria cases and 16,733 related deaths in 2017 in India. ${ }^{2}$ In India, malaria is more prevalent in Orissa accounting for $25 \%$ followed by Chhattisgarh (13\%), West Bengal(11\%), North Eastern States (10\%), Jharkhand(7\%) and Karnataka (7\%). ${ }^{3}$ The most severe clinical form of malaria is caused by P. falciparum infection. ${ }^{4}$ The disease is usually fatal in non-immunes. Falciparum malaria may be fatal if treatment is delayed beyond $24 \mathrm{~h}$ after the onset of clinical symptoms. ${ }^{5}$ The complications of falciparum malaria are cerebral malaria, pulmonary oedema, septicaemia and shock. ${ }^{6}$ If these complications are not treated adequately the patient becomes fatal. To prevent these complications, we need early diagnosis. Delayed diagnosis and comatose condition were the main determinants of death. Clinical diagnosis of malaria requires microscopically observation of the parasite. Diagnosis of malaria by microscopy is a gold standard but requires expertise, consumes time. Recently, a new rapid malaria detection tests based on detection of an enzyme produced by live parasites, parasite lactate dehydrogenase (pLDH) and Histidine Rich proteins (HRPII). In the present study, the Rapid Diagnostic Tests are evaluated for the effectiveness for early diagnosis of Malaria in Rural India to prevent deadly complications.

The authors wanted to evaluate the effectiveness of Rapid Diagnostic Test (RDT) in early diagnosis of Malaria in Rural India to prevent deadly complications of Malaria.

\section{METHODS}

\section{Research Design}

The Study was hospital based cross sectional study conducted in a hospital included 733 patients with fever of 3 days duration. Fever with chills is included and the subjects with known history of HIV and Tuberculosis are excluded. After obtaining consent, 733 whole blood samples were collected and tested for Smear Microscopy for Malarial parasites and also tested with Rapid Diagnostic Tests. The results of RDT are compared with the results of Gold Standard technique i.e., peripheral smear for Malarial parasites with the help of $\mathrm{X}^{2}$ values and sensitivity, specificity, positive predictive values and negative predictive values, Percentage of False Negatives, Percentage of False Positives, Positive Likelihood Ratio(LR+), Negative Likelihood Ratio(LR-) values. The study included 733 patients with fever of several days' duration. Consent was taken for the study and sample collection. Ethical committee approval was obtained. The study was spread over 10 months from June, 2018 to March, 2019 and results were analysed.

\section{Statistical Techniques}

High sensitive test is one that correctly identifies patients with a disease, also called true positive rate. High specificity test is one that correctly identifies patients without disease, also called true negative rate. Positive Predictive Value indicates the probability of a disease in persons who have a positive test result. Negative Predictive Value indicates the probability of not having the disease in persons who have a negative test result. Chi-square is applied to test the goodness of fit to verify the distribution of observed data with assumed theoretical and expected frequencies. A null hypothesis is established and verified with Chi-square test.

\section{RESULTS}

Comparison of Smear Microscopy and Rapid Diagnostic Test in the Diagnosis of Malaria

In Diagnosis of Malaria, Rapid Diagnostic Test has shown Sensitivity value as $97.81 \%$, Specificity value as $97.82 \%$, Positive predictive value as $97.20 \%$ and Negative predictive value as $98.30 \%$. Percentage of False Negatives as 2.19, Percentage of False Positives as 2.18, Positive Likelihood Ratio (LR+) 44.89, Negative Likelihood Ratio (LR-) 0.02. At Degrees of freedom 1 and at 0.05 significance level, the table value is 3.841 . The calculated value is 0.02 which is less than the table value. Hence the null hypothesis, "There is no difference in the diagnosis of malaria between smear microscopy and Rapid Diagnostic Test' is retained. Comparison data shows Rapid Diagnostic Test is as effective as Smear for MP in diagnosis of Malaria.

\begin{tabular}{|c|c|c|c|}
\hline Blood Smear & \multirow{2}{*}{ Positive } & \multirow{2}{*}{ Negative } & \multirow{2}{*}{ Total } \\
\hline RDT & 313 & 9 & 322 \\
\hline Positive & 7 & 404 & 411 \\
\hline Negative & $\mathbf{3 2 0}$ & $\mathbf{4 1 3}$ & $\mathbf{7 3 3}$ \\
\hline Total & 97.81 \\
\hline \multicolumn{2}{|c|}{ Sensitivity } & 97.82 \\
\hline \multicolumn{2}{|c|}{ Positive Predictive Value } & 97.20 \\
\hline Negative Predictive Value & 98.30 \\
\hline Percentage of False Negatives & 2.19 \\
\hline Percentage of False Positives & 2.18 \\
\hline Positive Likelihood Ratio (LR+) & 0.02 \\
\hline Negative Likelihood Ratio (LR-) \\
Table 1. Calculation of Sensitivity and Specificity of Rapid \\
Diagnostic Test in the Diagnosis of Malaria \\
\hline
\end{tabular}

\begin{tabular}{|c|c|c|c|c|c|c|c|c|c|c|}
\hline \multirow[b]{2}{*}{ Test } & \multicolumn{5}{|c|}{ Positive } & \multicolumn{5}{|c|}{ Negative } \\
\hline & 0 & 되 & 突 & ָْ & 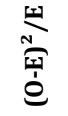 & 0 & 四 & อ̊ & ْ & 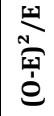 \\
\hline Blood Smear & 320 & 320 & 0 & 0 & 0.00 & 413 & 413 & 0 & 0 & 0.00 \\
\hline RDT Test & 322 & 320 & 2 & 4 & 0.013 & 411 & 413 & -2 & 4 & 0.010 \\
\hline \multicolumn{10}{|c|}{$\Sigma \mathrm{x}^{2}=$} & 0.02 \\
\hline
\end{tabular}

Table 2. Chi-Square Estimation between Smear Microscopy and Rapid Diagnostic Test in the Diagnosis of Malaria

Comparison of Smear Microscopy and Rapid Diagnostic Test in the Diagnosis of Falciparum Malaria

In diagnosis of Falciparum Malaria, Rapid Diagnostic Test has shown Sensitivity value as $97.98 \%$, Specificity value as 98.79\%, Positive predictive value as $97.49 \%$ and Negative predictive value as $99.03 \%$. Percentage of False Negatives as 2.02, Percentage of False Positives as 1.21, Positive Likelihood Ratio (LR+) 80.93, Negative Likelihood Ratio (LR-) 0.02. At Degrees of freedom 1 and at 0.05 significance level, the table value is 3.841 . The calculated value is 0.01 is less than the table value. Hence the null hypothesis, "There is no difference in the diagnosis of Falciparum malaria between smear microscopy and Rapid Diagnostic Test' is retained. 
Comparison data shows Rapid Diagnostic Test is as effective as Smear for MP in diagnosis of Falciparum Malaria.

\begin{tabular}{|c|c|c|c|}
\hline Blood Smear & \multirow{2}{*}{ Positive } & \multirow{2}{*}{ Negative } & \multirow{2}{*}{ Total } \\
\hline RDT & 194 & 5 & 199 \\
\hline Positive & 4 & 408 & 412 \\
\hline Negative & $\mathbf{1 9 8}$ & $\mathbf{4 1 3}$ & $\mathbf{6 1 1}$ \\
\hline Total & & 97.98 \\
\hline \multicolumn{2}{|c|}{ Sensitivity } & 98.79 \\
\hline Positive Predictive Value & 97.49 \\
\hline Negative Predictive Value & 99.03 \\
\hline Percentage of False Negatives & 2.02 \\
\hline Percentage of False Positives & 1.21 \\
\hline Positive Likelihood Ratio (LR+) & 00.93 \\
\hline Negative Likelihood Ratio (LR-) & 0.02 \\
\hline Table 3. Calculation of Sensitivity and Specificity of Rapid \\
Diagnostic Test in the Diagnosis of Falciparum Malaria \\
\hline
\end{tabular}

\begin{tabular}{|c|c|c|c|c|c|c|c|c|c|c|}
\hline \multirow[b]{2}{*}{ Test } & \multicolumn{5}{|c|}{ Positive } & \multicolumn{5}{|c|}{ Negative } \\
\hline & 0 & 디 & 仓̊ํ & 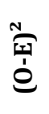 & 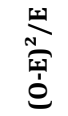 & 0 & 되 & 仓이 & 菌 & 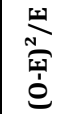 \\
\hline Blood Smear & 198 & 198 & 0 & 0 & 0.00 & 413 & 413 & 0 & 0 & 0.00 \\
\hline RDT Test & 199 & 198 & 1 & 1 & 0.005 & 412 & 413 & -1 & 1 & 0.002 \\
\hline \multicolumn{10}{|c|}{$\Sigma \mathbf{x}^{2}=$} & 0.01 \\
\hline
\end{tabular}

Comparison of Smear Microscopy and Rapid Diagnostic Test in the Diagnosis of Vivax Malaria

In diagnosis of Vivax malaria, Rapid Diagnostic Test has shown Sensitivity value as $97.54 \%$, Specificity value as $99.03 \%$, Positive predictive value as $96.75 \%$ and Negative predictive value as $99.27 \%$. Percentage of False Negatives as 2.46, Percentage of False Positives as 0.97, Positive Likelihood Ratio (LR+) 100.71, Negative Likelihood Ratio (LR-) 0.02. At Degrees of freedom 1 and at 0.05 significance level, the table value is 3.841 . The calculated value is 0.01 is less than the table value. Hence the null hypothesis, "There is no difference in the diagnosis of Vivax malaria between smear microscopy and Rapid Diagnostic Test' is retained. Comparison data shows Rapid Diagnostic Test is as effective as Smear for MP in diagnosis of Vivax Malaria.

\begin{tabular}{|c|c|c|c|}
\hline Blood Smear & \multirow{2}{*}{ Positive } & \multirow{2}{*}{ Negative } & \multirow{2}{*}{ Total } \\
\cline { 1 - 3 } RDT & 119 & 4 & 123 \\
\hline Positive & 3 & 409 & 412 \\
\hline Negative & $\mathbf{1 2 2}$ & $\mathbf{4 1 3}$ & $\mathbf{5 3 5}$ \\
\hline Total & & 97.54 \\
\hline \multicolumn{2}{|c|}{ Sensitivity } & 99.03 \\
\hline Positive Predictive Value & 96.75 \\
\hline Negative Predictive Value & 99.27 \\
\hline Percentage of False Negatives & 2.46 \\
\hline \multicolumn{2}{|c|}{ Percentage of False Positives } & 0.97 \\
\hline Positive Likelihood Ratio (LR+) & 100.71 \\
\hline Negative Likelihood Ratio (LR-) & 0.02 \\
\hline Table 5. Calculation of Sensitivity and Specificity of Rapid \\
Diagnostic Test in Diagnosis of Vivax Malaria \\
\hline
\end{tabular}

\begin{tabular}{|c|c|c|c|c|c|c|c|c|c|c|}
\hline \multirow[b]{2}{*}{$\mathbf{z}$} & \multicolumn{5}{|c|}{ Positive } & \multicolumn{5}{|c|}{ Negative } \\
\hline & 0 & 四 & @્a & 茨 & 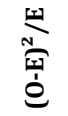 & 0 & [1 & อ̊ & 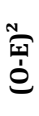 & 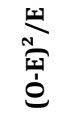 \\
\hline Blood Smear & 122 & 122 & 0 & 0 & 0.00 & 413 & 413 & 0 & 0 & 0.00 \\
\hline RDT Test & 123 & 122 & 1 & 1 & 0.008 & 412 & 413 & -1 & 1 & 0.002 \\
\hline \multicolumn{10}{|c|}{$\Sigma \mathrm{x}^{2}=$} & 0.01 \\
\hline
\end{tabular}

\section{DISCUSSION}

The present study compared Rapid Diagnostic Tests with Smear Examination as gold standard and concluded RDT with high sensitivity 97.81\%, high specificity $97.82 \%$, Positive predictive value as $97.20 \%$ and Negative predictive value as 98.30\%. Percentage of False Negatives as 2.19, Percentage of False Positives as 2.18, Positive Likelihood Ratio (LR+) 44.89, Negative Likelihood Ratio (LR-) 0.02. In diagnosis of Falciparum malaria also, Rapid Diagnostic Test has shown

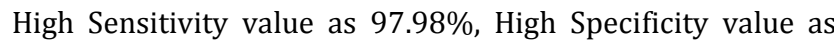
98.79\%, Positive predictive value as $97.49 \%$ and Negative predictive value as $99.03 \%$. Percentage of False Negatives as 2.02, Percentage of False Positives as 1.21, Positive Likelihood Ratio (LR+) 80.93, Negative Likelihood Ratio (LR) 0.02. In differentiating Vivax malaria, Rapid Diagnostic Test

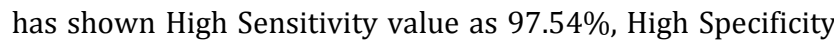
value as $99.03 \%$, Positive predictive value as $96.75 \%$ and Negative predictive value as $99.27 \%$. Percentage of False Negatives as 2.46, Percentage of False Positives as 0.97, Positive Likelihood Ratio (LR+) 100.71, Negative Likelihood Ratio (LR-) 0.02.

Sensitivity refers to the test's ability to correctly detect ill patients who do have the condition. A high sensitivity test is reliable, since it rarely misdiagnoses those who have the disease. A negative test result would definitively rule out presence of the disease in a patient. Specificity relates to the test's ability to correctly reject healthy patients without a condition. The test with high specificity rarely gives positive results in healthy patients. A test with a higher specificity has a lower type I error rate. Likelihood Ratio (LR+) Values greater than 1 increase the probability of disease. Likelihood Ratio (LR-) Values between 0 and 1 decrease the probability of disease. As Rapid Diagnostic Test has shown high Sensitivity and high specificity, high Likelihood Ratio (LR+) and Likelihood Ratio (LR-) values less than 0 in diagnosis of malaria and differentiating the malarial species, it can be considered as good screening test for the diagnosis of malaria and also malarial species differentiation.

At Degrees of freedom 1 and at 0.05 significance level, Chi-Square estimations are less than the table value 3.841 . Hence the hypotheses "There is no difference in the diagnosis of malaria between smear microscopy and Rapid Diagnostic Test', 'There is no difference in the diagnosis of Falciparum malaria between smear microscopy and Rapid Diagnostic Test' and 'There is no difference in the diagnosis of Vivax malaria between smear microscopy and Rapid Diagnostic Test' are retained. Hence it is concluded that Rapid Diagnostic Tests is as effective as Smear for Malarial parasite examination which is gold standard in diagnosis of malaria and differentiating the malarial species.

The data of the present study coincides with the previous studies. Parija SC et $\mathrm{al}^{7}$ in their study compared thick smear as gold standard with RDT shown sensitivity of $75 \%$. Bhat Sandhya $\mathrm{K}$ et $\mathrm{al}^{8}$ in their study, compared RDT with thick smear as gold standard and shown RDT sensitivity, specificity of $95.45 \%, 100 \%$, respectively. Bhavnaben A. Vora et al $^{9}$ in their comparative study, Peripheral smear had $85.5 \%$ sensitive and $100 \%$ specific compared to RDT which was 100 $\%$ sensitive and specific whereas centrifuged buffy coat was $92.7 \%$ sensitive and $99.3 \%$ specific. Samina Naz Mukry et al ${ }^{10}$ 
concluded in their study that RDT with a sensitivity of $91.52 \%$. Manali M. Kocharekar et al ${ }^{11}$ studied the efficacy of RDT and concluded the sensitivity of Parahit total, Advantage mal card, and modified QBC for P. falciparum detection was $70.3 \%, 95 \%$, and $98 \%$, and specificity was $98 \%, 98 \%$, and $96 \%$, respectively. Saha $\mathrm{S}$ et al ${ }^{12}$ concluded in their study that the sensitivity and specificity of microscopy was $63 \%, 99.4 \%$ respectively. Sensitivity and specificity of RDT was $94 \%$ and 99\% respectively. RDT had the highest LR for positive test result 175 and the lowest LR for negative test result 0.058. SS Sahu et al $^{13}$ concluded in their study to improve the efficiency (Sensitivity) of the kit so that the true falciparum infections will not be missed especially in areas where P. falciparum has been the predominant species causing cerebral malaria with the results of the sensitivity of ParaHIT-f was $63.6 \%$ and specificity was $98.9 \%$. With positive and negative predictive values (PPV and NPV) of $92.6 \%$ and $93.0 \%$ respectively. NitiN Joseph et al ${ }^{14}$ stressed in their study that Diagnostic Antigen detecting MRDT showed good performance as a screening test and hence can be recommended for wide-scale usage at these settings with sensitivity of $98.6 \%$, specificity of $86.9 \%$, Positive Predictive Value (PPV) of $94.1 \%$ and Negative Predictive Value (NPV) of $96.6 \%$. The study by Falade et al ${ }^{15}$ concluded that

RDTs are useful in guiding malaria management and were successfully used for diagnosis by trained CHWs. Sensitivity and specificity of SD-Bioline RDT were $94.3 \%$ and $41.6 \%$ respectively, while the negative and positive predictive values were $86.1 \%$ and $65.6 \%$, respectively. Gupta et al ${ }^{16}$ concluded that the RDT based on malaria antigen method is as specific and sensitive as the traditional PBS microscopy with Sensitivity and specificity of RDT were $91.8 \%$ and 93.8\%, respectively. Positive predictive value and negative predictive value were $97.8 \%$ and $98.9 \%$ respectively. Batwalaet al 17 compared the RDT with smear for microscopy and concluded with the results of the sensitivity of RDT and Microscopy $91.0 \%$, 46\% respectively. The specificity of RDT and Microscopy were $86.3 \%, 93.4 \%$ respectively. Ayogu E E ${ }^{18}$ concluded that Rapid diagnostic tests could be useful in areas with high parasite density as an alternative to smear microscopy with the sensitivity and specificity PPV and NPV of RDT $82.2 \%, 100.0 \%, 100.0 \%$, and $34.3 \%$, respectively. Oyetunde $\mathrm{T}$ et $\mathrm{al}^{19}$ in their study considering microscopy as the gold standard, RDT exhibited high specificity $87.1 \%$ and low sensitivity $42.5 \%$ with positive predictive and negative predictive values of 86.6 and $43.5 \%$, respectively. Jaswinder Sharma et al ${ }^{20}$ concluded in their study that the sensitivity of RDT is $97.4 \%$, specificity $100 \%$ when compared to microscopy.

\section{CONCLUSIONS}

The Rapid Diagnostic Tests are effective screening tests. Comparison with Smear Microscopy has shown that Rapid Diagnostic Tests have high sensitivity, specificity and predictive values, high Likelihood positive (LR+) and low Likelihood Negative (LR-) values. The rapid test is simple, cheap and reliable tool to diagnose malaria early to prevent deadly complications, especially in rural and tribal areas where facilities are insufficient.

\section{REFERENCES}

[1] World Malaria 2018 https://www.who.int/malaria/publications/worldmalaria-report-2018/report/en/

[2] World Malaria Report 2018. https://www.businessstandard.com/article/current-affairs/world-malariareport-2018-led-by-odisha-india-reduces-cases-by-3million-118112300064_1.html

[3] Kumar A, Valecha N, Jain T, et al. Burden of malaria in India: retrospective and prospective view. Am J Trop Med Hyg 2007;77(Suppl 6):69-78.

[4] Chen Q, Schlichtherle M, Wahlgren A. Molecular aspects of severe malaria. Clinical Microbiology Reviews 2000;13(3):439-50.

[5] https://www.who.int/ith/diseases/malaria/en/

[6] Trampuz A, Jereb M, Muzlovic I, et al. Clinical review: severe malaria. Crit Care 2003;7(4):315-23.

[7] Parija SC, Dhodapkar R, Elangovan S, et al. A comparative study of blood smear, QBC and antigen detection for diagnosis of malaria. Indian Journal of Pathology \& Microbiology 2009;52(2):200-2.

[8] Bhat SK, Sastry AS, Nagaraj ER, et al. Laboratory diagnosis of malaria by conventional peripheral blood smear examination with Quantitative Buffy Coat (QBC) and Rapid Diagnostic Tests (RDT) - a comparative study. International Journal of Collaborative Research on Internal Medicine \& Public Health 2012;4(10):1746-55.

[9] Vora BA, Bindu RS. Comparison of diagnostic methods of malaria by peripheral smear, centrifuged buffy coat smear and rapid antigen detection test. International Journal of Research in Medical Sciences 2017;10:4532-7.

[10] Mukry SN, Saud M, Sufaida G, et al. Laboratory diagnosis of malaria: comparison of manual and automated diagnostic tests. Canadian Journal of Infectious Diseases and Medical Microbiology 2017;2017:9286392.

[11] Kocharekar MM, Sarkar SS, Dasgupta D. Comparative study of modified quantitative buffy coat and two rapid tests in comparison with peripheral blood smear in malaria diagnosis in Mumbai, India. Article ID 194651, Journal of Parasitology Research 2014;2014:7. http://dx.doi.org/10.1155/2014/194651

[12] Saha S, Narang R, Deshmukh P, et al. Diagnostic efficacy of microscopy, rapid diagnostic test and polymerase chain reaction for malaria using bayesian latent class analysis. Indian J Med Microbiol 2017;35(3):376-80.

[13] Sahu SS, Gunasekaran K, Jambulinga P. Field performance of malaria rapid diagnostic test for the detection of Plasmodium falciparum infection in Odisha state, India. Indian Journal Medical Research 2015;(Suppl 142):S52-8.

[14] Nitin J, AdityA KU. Validation of malaria antigen detecting rapid diagnostic test kit: a study from highly endemic area in Coastal India. Journal of Clinical and Diagnostic Research 2018;12(9):LC16-LC20. 
[15] Falade CO, Ajayi IO, Nsungwa-Sabiiti J, et al. Malaria rapid diagnostic tests and malaria microscopy for guiding malaria treatment of uncomplicated fevers in Nigeria and pre-referral cases in 3 African Countries. Clinical Infectious Diseases 2016;63(Suppl 5):290-S7.

[16] Gupta P, Gupta P, Rao S, et al. Comparison between microscopy and rapid diagnostic tests in diagnosis of malaria at a tertiary care medical institution in Uttarakhand (a 3-year study). Asian J Pharm Clin Res 2018;11(2):94-6.

[17] Batwala V, Magnussen P, Nuwaha F. Are rapid diagnostic tests more accurate in diagnosis of plasmodium falciparum malaria compared to microscopy at rural health centres? Malaria Journal 2000;9:349.
[18] Ayogu EE, Ukwe CV, Nna EO. Assessing the reliability of microscopy and rapid diagnostic tests in malaria diagnosis in areas with varying parasite density among older children and adult patients in Nigeria. J Postgrad Med 2016;62(3):150-6.

[19] Oyeyemi OT, Ogunlade AF, Oyewole IO. Comparative assessment of microscopy and rapid diagnostic test (RDT) as malaria diagnostic tools. Research Journal of Parasitology 2015;10(3):120-6.

[20] Sharma J, Singh S, Kaur A, et al. Comparative evaluation of microscopic and rapid diagnostic test (RDT) for diagnosis of malaria in a tertiary care hospital in Bathinda. Int J Health Sci Res 2017;7(6):90-3. 